\title{
Learning Conversations in World of Warcraft
}

\author{
Bonnie A. Nardi, Stella Ly, and Justin Harris \\ University of California, Irvine \\ forthcoming in Proc. HICSS 2007
}

\begin{abstract}
We examine learning culture in a popular online game, World of Warcraft. We analyze the way players learn this complex game through chat conversation with peers. We describe three kinds of learning: fact finding, devising tactics/strategy, and acquiring game ethos. We investigate learning in the zone of proximal development as specified in cultural-historical activity theory. We examine the emotional tenor of learning conversations, noting their drama, humor, and intimacy.
\end{abstract}

\section{Introduction}

World of Warcraft is one of the most popular online video games, with 6.5 million active subscribers in North America, Asia, and Europe [1]. Produced by Blizzard Entertainment, it is a massively multiplayer online game (MMOG) in which players are connected through the Internet in persistent worlds. Players develop characters that explore, fight, socialize, make money, take up professions, and advance through 60 levels of play. Play is complex, requiring the development of strategy, discovery of thousands of game facts, and subtle choices about character development [see 4, 12, 13 on the complexity of some video games]. Despite this, the manual that comes with the game is a slim 4 x 5 inch volume. No teachers, coaches, or curriculum explain the game. None of the familiar supports of formal education are in evidence - but no one fails World of Warcraft.

We have been engaged in ongoing ethnographic fieldwork in "WoW" since late 2005. The fieldwork is comprised of participation in the game, in-depth interviews (face-to-face and online), the collection of chat logs, and reading documents such as WoWrelated forums and websites.

Our research is guided by Vygotsky's notion of the zone of proximal development. Vygotsky understood learning as a social practice situated in the zone of proximal development in which a learner advances by taking on a challenge and using resources supplied by a teacher or more experienced peers to meet the challenge. The zone is the difference between what the learner can do with and without the aid of the teacher or peers [15]. Our analysis focuses on peer learning. Vygotsky specified "more experienced peers" in his formulation. For convenience, we will simply refer to "peers" assuming that if they can instruct they are more knowledgeable, unless otherwise noted.

We attempt to unpack what happens in the zone of proximal development (ZPD) as players learn the game. Vygotsky sketched a general notion of the zone of proximal development without developing it in detail [9]. Therefore empirical accounts of what transpires in the ZPD are needed. Many excellent accounts have been provided [see 9] but the vast majority involve learning based on a defined curriculum as a planned set of learning experiences. Such a curriculum might be a traditional school-based curriculum, or the more informal "learning curriculum" [7] or "situated curriculum" [5]. Our goal in this paper is to describe learning activity that is devoid of curriculum. We examine learning enacted in spontaneous chat conversations initiated by participants pursuing their own playful objectives.

Although we utilized the ZPG in this analysis, we also followed a grounded theory approach [6] to identify key areas of learning in World of Warcraft. This analysis uncovered three key areas: fact finding, tactics and strategy, and game ethos. By ethos we mean "the distinguishing character, sentiment, moral nature, or guiding beliefs" [8] apparent in the game. Through conversation, players acquire basic knowledge needed to play the game, guidance on tactics and strategy, and initiation into the moral order or ethos of the game.

Chat conversation is a key means of learning in World of Warcraft but not the only means. Players also make use of player-created content on the Internet in forums, FAQs, guides, and commentaries. We do not have room in the scope of this paper to analyze those materials but 
recognize that they are important [see 14]. As in any endeavor, players also learn simply by doing. And players observe other players, learning from their successes and failures. In this paper we focus on learning in conversation, examining chat conversations among peers to see how such conversations enable learning.

\section{World of Warcraft}

Based on a high fantasy motif, World of Warcraft is a role-playing game in which players create characters with distinctive looks and abilities. The player controls an animated character in a visually complex world of forests, farms, oceans, deserts, mountains, and swamps, as well as several major cities, small towns, and wilderness encampments. Activities include battling monsters, exploration, and "quests," or missions, in addition to crafting, chatting, and traveling to cities for banking, training, and trading. Most players join guilds which are named groups, so there are others with whom to socialize and play. Players may belong to only one guild at a time. Many players have more than one character-a "main" and "alts" or alternative characters. Only one character is played during a login.

The quest is an important feature of the game. Each quest has a name, a goal, and a reward. Questing usually involves battling monsters. The goal may require slaying a certain number of evil creatures; obtaining items such as a document, key, or reagent; or delivering messages or supplies. Players can assemble temporary formal groups for questing including parties (up to five players) or raids (up to forty players).

Game money may be earned as a quest reward or claimed from slain monsters. Money can also be earned through trade with both computer- and player-controlled characters. Money is used to purchase items such as armor, weapons, and reagents for things like making potions or creating enchantments.

Even beyond the guild, WoW is a very social game and players are always aware of other players. Characters are labeled with their name and guild, and mousing over the character reveals class and level. This information is color coded so enemies in the opposing "faction" are apparent. The information helps players know something about how they might interact with players they don't know; for example, can they ask for help, suggest a duel, or initiate player vs player combat?
WoW is a richly "chatful" environment with several channels scoped to different geographies, activities, and social relationships. Channels are available for guilds, parties, and raids. There are specific regional channels for forming parties and raids, and trading. A serverwide channel enables general comments and questions. Users can create their own chat channels. There is a channel limited to a local radius around the character analogous to the character speaking out loud. Players can chat privately in "whispers." In all forms of chat except whispers, anyone with access to the channel can view the conversation. These players can be reading the chat and making note of what they learn for future reference even if they are not actively chatting. Chat is thus a community resource for learning as well as enabling learning by those actually chatting.

\subsection{Learning WoW}

Players learn astonishingly detailed content in World of Warcraft. The game has eight "races" and "nine" classes. The races are Night Elf, Dwarf, Gnome, Human, Troll, Orc, Tauren, and Undead. Classes are druid, priest, warrior, rogue, mage, hunter, paladin, shaman, and warlock.

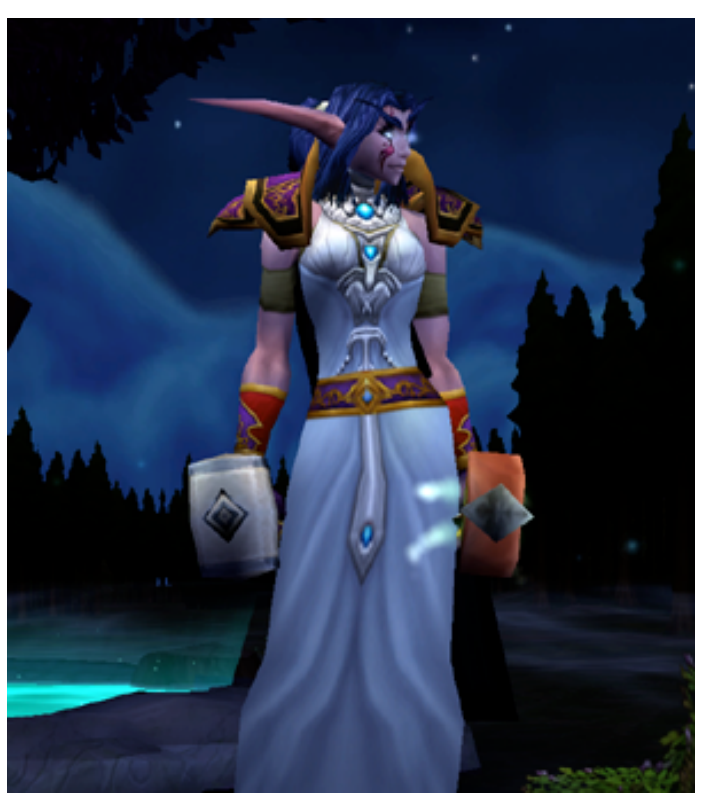

Figure One a night elf priest 
As a member of the race of Night Elves, the character pictured above has certain innate characteristics. As a member of the class of priests, she can develop abilities to heal the wounded, cure disease, resurrect characters that have died in battle, read the minds of enemies, and fight. The use of each ability requires precise timing based on monitoring several variables that determine when to act. The choice of ability involves understanding the monsters she is battling as well as the abilities of the other classes with whom she is playing.

Each character can acquire two professions. This priest is an herbalist and alchemist. She needs considerable knowledge to practice her professions. Where can rare herbs be found? Which potions are useful for which classes? How should she price her goods for sale to other players? At a minimum, players must know the key abilities of each of the nine classes, their own armor and weapons, skills needed for professions, the complex game geography, how to assign "talent points" to make the character powerful, how class-specific quantities such as "mana" and "health" are handled, how to position the character for maximum effectiveness in battle, and when to run away!

\section{Methods}

Our research is based on participantobservation fieldwork. Each of us created at least two characters and joined at least one guild. We have jointly played for over 25 months and continue to play. Chat logs were collected during our game play using a function supplied in the game. Orthography in chat transcripts reported here is unaltered. All character names are pseudonyms. We conducted 30 in-depth interviews (some online and some face to face) and read the many forums, FAQs, guides, commentaries, and other materials devoted to World of Warcraft. Our analysis focuses on chat logs but is informed by what we learned in the interviews and during play. The research generated thousands of pages of transcripts. This paper focuses on in-depth analysis of several conversations from the corpus.

\section{Learning in conversation}

In World of Warcraft, learning in conversation is event-driven with no planned curriculum. It is spontaneous, erratic, serendipitous, and contextual. It is something like Gherardi's situated curriculum [5]. However, the situated curriculum comprises a sequence of tasks for students to complete with appropriate instruction as the student engages in the tasks. In WoW, learning in conversation is driven by small events such as players asking questions or receiving advice during play.

We examine the emotional tenor of learning conversations, noting their drama, humor, and intimacy. We believe emotion is present in many learning conversations, whether on- or offline, but that it remains unexamined as a potential learning resource. A goal of this paper is to point out the presence of emotion in learning conversations, to focus beyond the informational content of the conversations, and to suggest emotion as an important topic for future research [see 11].

\subsection{Fact finding}

Much learning in WoW is in the form of simple facts such as where a computer character is, what can be done with a certain item, or which professions can provide particular services. Players find facts in several ways. They ask questions as they solve problems for particular activities. They acquire facts as they come up in chat erratically and unpredictably. They learn facts when other players observe deficiencies in their actions and tell them what to do.

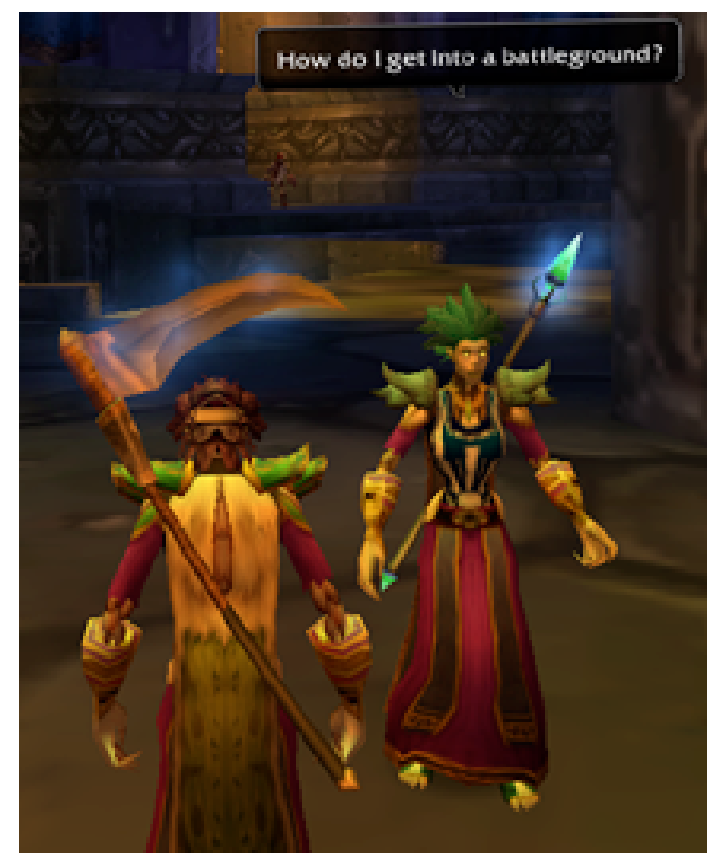

Figure Two. Finding a battleground 
Many fact finding questions are very simple. For example, in Figure 2 one player asks how to get to get into a "battleground," an area where teams engage in contests such as capture the flag. Players amass thousands of game facts through these simple exchanges. They provide fast answers to informational queries so players can keep moving through the game unimpeded by lack of knowledge.

Guild chat is a rich source for fact finding. In the following conversation, a player named Drollnar offers an item that he doesn't need to any guild member who might want it.

[Guild] Drollnar: Hey any drinkers do you want this Volatile Rum?

[Guild] Akiraa: me

[Guild] Miggles: no don't drink ...expensive for alchamy

[Guild] Physikz: lol ME ME ME!

[Guild] Miggles: sell in ah [Auction House] for alot of money

[Guild] Drollnar: is it a trade good

[Guild] Miggles: yes need it to make goblin rocket fuel

[Guild] Drollnar: well u learn some thing new every day

[Guild] Miggles: good thing to [have] for money...don't drink it lol

[Guild] Drollnar: well sorry i am going 2 have to sell this but $\mathrm{i}$ will send both poeple that want ed it something nice soon

Miggles reveals that the Volatile Rum can be sold. He explains that it is an expensive ingredient useful for the practice of alchemy. He observes that the item can be sold in the Auction House for a good price. He first issues a direct instruction with a rationale, telling Drollnar not to sell the rum because of its status as an expensive ingredient. He then notes the way in which Drollnar might dispose of the rum at the Auction House. Finally Miggles observes that Volatile Rum is used to make goblin rocket fuel. The instruction is entirely casual but informative, examining the rum from several points of view.

Drollnar signals that he has learned what to do with the rum by remarking, "well u learn some thing new every day." Miggles's next utterance caps the instruction through repetition; the item has monetary value and should not be drunk. Miggles adds "lol" to suggest friendly intention since he has given another direct order but he knows that Drollnar understands. Lol stands for "laugh out loud." It is often used to denote affection or playfulness in a comment that might otherwise sound rude.

There is a little tension in this exchange because Drollnar decides to withdraw his request to offer the rum, given what he's learned. He will sell the rum but he promises Akiraa and Physikz "something nice soon." Unlike learning facts by rote memory in school, players often acquire facts through the sociality of mini-dramas that take place in chat. The exchange about the rum is the opposite of a set of decontextualized facts in several ways. The information Drollnar received was tailored to his level of knowledge, it occurred in a social context, offered an action for him to take to further his own ends, and took meaning from the participation of other players who instructed (Miggles) or injected statements of desire into the discourse (Akiraa and Physikz).

We thus observe something in the zone of proximal development that we have not seen described in other analyses - the presence of emotion. We believe it is likely that such emotion provides salience to the learned facts, heightens attention, and adds interest. Our current data do not allow us to delve further into such questions, but suggest the need for future research. Such research might focus on the way the zone of proximal development shapes learning not only through helping learners make new connections and acquire deeper understandings, but by providing a supportive, lively environment in which to learn. In this conversation, the interest expressed by Akiraa and Physikz in the Volatile Rum is likely to have made it seem more important and salient to Drollnar, while Miggles's proactive interest provided positive support.

Learning about the Volatile Rum was serendipitous and spontaneous; Drollnar did not know he was about to receive instruction. Miggles seized the moment to help Drollnar dispose of the rum in an advantageous way. (We can vouch for the fact that others learned from this exchange because one of the authors is an alchemist now hoarding Volatile Rum.)

Learning in conversation may take place in combat when players are working closely together in the same game space, with the same objectives. In such circumstances players may tell others (often in no uncertain terms) how to play. The possibility of death for any player, even a "wipe" in which every player dies, is at stake. In the following chat, one player instructs another during battle. They are in a "pick-up" party and do not know each another. 
[Party] Zoros: use sunder armor to take aggro from us cold

[Party] Coldnight: i don't have sunder armor

[Party] Zoros: u should lol

Zoros tells Coldnight to use an ability possessed by the warrior class, sunder armor, which will attract the enemies' attention ("aggro" or aggression). The job of a warrior is to draw the aggro and take the damage while others attack. Coldnight does not yet have the sunder armor ability although his level indicates to Zoros that he should. She tells him to obtain the ability, but softens the suggestion with lol. In this context, lol clearly does not mean "laugh out loud" since nothing particularly funny has been said. Zoros makes her instruction friendly by inventing the nickname "cold" for Coldnight. These practices are ubiquitous in WoW where fast-paced action and the risk of players taking offense at briefly worded commands are mitigated by such rhetorical conventions. After this experience, Coldnight set out on a series of quests specific to warriors in which he obtained sunder armor, enabling him to play his class much more effectively.

This learning conversation contains an element of drama-Zoros is worried about dying in battle as the party is being attacked by multiple ferocious Dark Iron Dwarves. The conversation was event-driven, timed to deliver a key message to a player in need of an important ability.

\subsection{Tactics and strategy}

Players must do more than amass facts. They need to understand at a deeper level how to play their character. Acquaa is trying to learn how to pit her warlock against every other class in the game for player versus player combat in which players battle other players instead of computer characters. She asks a nearby player, Hakiro, a rogue, to tell her how to beat rogues.

[Party] Acquaa: tell me what are rogues weaknesses... like how can i do well against them?

[Party] Hakiro: void walker

[Party] Acquaa: why?

[Party] Hakiro: sacrifise him to get bubble

[Party] Acquaa: i hate doing that though

[Party] Hakiro: it saves ur!@\#

[Party] Acquaa: the bubble dodsn't last for long

[Party] Hakiro: everytime i got wooped by a lock he used bubble
[Party] Hakiro: just fear them

[Party] Acquaa: fear, dot dot dot, then RUNNNNNN

Warlocks are spellcasters whose delicate armor provides little protection. Acquaa reported that she disliked rogues since they so often attack classes with weak armor. Her desire to know how to fight them was motivated by her emotional response to rogues' tactics. Rogues sneak around invisibly, stab characters in the back, and hit hard and fast. Acquaa needs to know how to exploit their weaknesses.

Hakiro merely says "void walker" when Acquaa asks her carefully worded question. She is unsure what he means. Hakiro explains that by "sacrificing" the void walker (removing him) she will get a "bubble" - a protective shield the rogue cannot break through. Acquaa is doubtful because the bubble doesn't last very long and she will lose her pet. But Hakiro speaks from experience-every time he has been "wooped" it was by a warlock who used the bubble. Hakiro adds the next step in the procedure, to fear the opponent. Acquaa sees where that is going. She understands the rest of the sequence and completes it herself. "Dot" refers to casting slow spells (damage over time) when the rogue is feared. She will then have to end by running away, but her spells will still be delivering damage, ultimately defeating the rogue. Acquaa reported that after this exchange she was much better at fighting rogues.

This conversation is characterized by a kind of casual intimacy common in World of Warcraft. Acquaa and Hakiro do not know each other well, but they fall into an easy rhythm of expressive question and answer. Hakiro admits past defeats (when he was "wooped") and Acquaa pushes him to explain exactly what she must do and why. Hakiro uses humor ("it saves ur !@\#”) and Acquaa rejoins with the witty "fear, dot dot dot, then RUNNNNNN." Just as drama enlivened the learning experience in some of our previous examples, casual intimacy colors this one.

Learners at the same level may jointly discover new knowledge through conversation. Ixchelle and Laizza are searching for an Undead courier they must find to retrieve a document he is carrying. Where is he? Many quests are vaguely worded and part of the game is to figure out what they mean. Ixchelle and Laizza have been searching fruitlessly for quite a while. They are near a house surrounded by Undead, but they 
are high level monsters, so Ixchelle and Laizza feel it cannot be the right place for their quest.

[Party] Ixchelle: maybe the courier is not inside that house but somewhere near

[Party] Laizza: you know, earleri today i got attacked by a bunch of lvl 35 undead guards walking on the path heading west

[Party] Ixchelle: i saw them and ran

[Party] Laizza: i just ran away and didnt look back

[Party] Ixchelle: :)

[Party] Laizza: maybe they have something to do with it?

[Ixchelle and Laizza come too close to the Undead near the house and are nearly killed before running away]

[Party] Laizza: LOL

[Party] Ixchelle: whew!

[Party] Laizza: we lived to fight another day!

[Party] Ixchelle: just barely

[Party] Laizza: lmao [laughing my ass off]

[Party] Ixchelle: any other ideas? i can't belive it's in that house

[they see the group of level 35 Undead walking down the road]

[Party] Laizza: i wonder if that squad is escorting the courier

[Party] Ixchelle: hmm. could be

Ixchelle and Laizza reason that the squad is escorting the courier. They return to town to seek help because the two of them can not manage the squad of five. Another player confirms that the squad is indeed protecting the courier. By reflecting in conversation on an observation Laizza made, and reasoning about the relative levels of the Undead at the house and in the squad, Ixchelle and Laizza arrived at the right conclusion about the location of the courier.

Stahl [13] observed that learning in small groups is characterized by a series of utterances that move the participants forward as each individual contribution builds on previous contributions. Ixchelle eliminates the house as a probable site for the courier which leads Laizza to suggest the roving squad. They are able to jointly produce the knowledge of the courier's location even though they are at the same level of experience, suggesting that the zone of proximal development may involve peers at the same level of experience in some cases.

This learning experience was heightened by a bit of drama and humor. Ixchelle and Laizza are attacked together and must run to escape. They joke about this, with Laizza exclaiming that they lived to fight another day. Again, emotion is part of the texture of learning.

\subsection{Game ethos}

Beyond fact finding and strategizing lie more subtle aspects of play. Players learn and invent the moral order of the game, coming to understandings about the right way to play. The moral order cannot be reduced to the simple acquisition of knowledge. It is in flux, under negotiation, emergent in conversation, and only temporarily stable. In the following conversation, a player's fact finding question becomes an occasion for reflecting on moral values. The players are discussing battlegrounds which are high stakes contests because calculations of "honor" points attaching to individual players are made.

[Guild] Vinius: If $\mathrm{u}$ leave a battle grounds early do $\mathrm{u}$ lose all your honor?

[Guild] Morninglight: should, deserter

[Guild] Morninglight: i've never left, too shameful

[Guild] Fiendish: I've left twice. Once by accident in my 3rd WSG [War Song Gulch Battleground] ever and once because I got D/C [disconnected]

Morninglight answers Vinius's factual query with a black-and-white moral judgment. Fiendish responds to Morninglight's assertion rather than answering Vinius's question. Morninglight's comment is more nuanced-he suggests there may be times when a player ends up leaving teammates in the lurch. In his own case, he left once by accident due to inexperience and once because he was disconnected. Fiendish does not disagree with Vinius but provides some concrete examples suggesting that not every desertion is "shameful." There is no direct confrontation yet differing moral perspectives are presented. The lesson in this conversation was serendipitous; Morninglight turned Vinius's factual question into a discussion of right and wrong in the game.

"Powerleveling" is a key issue in which the moral order of the game is contested. When powerleveling, a lower level player advances quickly through the 60 levels because higher level players accompany him, making the completion of quests much easier.

Player attitudes toward powerleveling vary. Some find it silly because why rush through the levels not really playing at all? Some believe it is ineffectual because lower level players do not learn to play on their own. Some take pride in accomplishing things on their own. Some find it acceptable practice because their goal is to arrive 
as quickly as possible at "endgame" activities available only to level 60 characters. Some guilds adopt powerleveling as a service they provide while others feel it is not in players' best interests.

In the following conversation, guildmates discuss the appropriateness of powerleveling, moving into a discussion of guild obligations. Janus is the guild master, a strong leader and influential figure in the guild. Recondo is somewhat older than the other players, the father of three.

Majimarg is joking that Janus's alternative character Golder should be kicked out of the guild because he has leveled so fast in a guild whose charter states that it is not a powerleveling guild. The joke leads to reflections in which the players consider what powerleveling is, its potential problems, and the larger role of the guild in helping players.

[Guild] Majimarg: even on the guild rules it says your not a powerleveling guild so go ahead and kick out golder

[Guild] Janus: powerleveling as such is permitted, we just dont provide it as a guild benefit

[Guild] Sartor: i started like a month ago and im lvl 34 and i didnt power lvl at all

[Guild] Recondo: No..but you did have a bit of help along the way Sartor

[Guild] Sartor: well yeah on qsts [quests]

[Guild] Sartor: thats what a guild is for helping others get lvled [leveled]

[Guild] Janus: yep, help, yes

[Guild] Kadnor: i think once u start a new character its easy cause u now [know] wat ur doin and were [where] 2 train

[Guild] Janus: but there is a subtle distinction between getting quest help, and being powerleveled (and not realy learning the game)

[Guild] Sartor: true

[Guild] Kadnor: iv done every quest in darny [a region of the game] by my self

[Guild] Sartor: but juss cuz u get powerlvled dont mean $u$ dont learn the game

[Guild] Janus: most of us are willing to help with quests. its the powerleveling we get problems with

[Guild] Sartor: yeah

[Guild] Recondo: What is your definition of power leveling, Janus?

[Guild] Janus: hard to say :)

[Guild] Sartor: powerlvling is when u get with a bunch or one high lvl and they take $u$ to high lvl training grounds

[Guild] Sartor: and u lvl off there [their] kills

[Guild] Recondo: Okay.,..don't mean to put you on the spot...this is a new discussion for me

[Guild] Janus: prob anything where you are just there to leach xp [experience points] repeatedly would be PL'ing.
[Guild] Recondo: I'd accept that Sartor

[Guild] Sartor: yeah ive played plenty of rpgs [role playing games] that pwrlvling is a major thing

[Guild] Janus: yep, it was big in EQ. but WoW has a lot of controls to prevent it

[Guild] Majimarg: what is EQ

[Guild] Majimarg: everquest nvm [never mind]

Recondo reminds Sartor that he had help from the guild. Recondo felt Sartor was overlooking this in commenting on how quickly he had leveled. Recondo's comment summons a moral obligation on Sartor's part to acknowledge the generosity of higher level players. Sartor rejoins, slightly defensively, by noting that the guild is supposed to help, calling forth the guild's obligation to serve. Janus agrees with Sartor, but uses the comment to propose a distinction between powerleveling and the kind of help Sartor received. Kadnor enters the conversation with another point of view, to make clear that not everyone gets help to level. He speaks tactfully, first noting that he has had more than one character and has learned the ropes. He firmly states, however, that he has successfully played independently.

Recondo is not done with the conversation; he wishes to define the moral order more precisely. He requests a definition of powerleveling from Janus. Janus is momentarily stumped but Sartor comes up with a clear definition. Janus responds with a more general formulation. Recondo finds this acceptable. He addresses his agreement to Sartor, signaling that he was calling Sartor on his boast and that the subsequent discussion has endorsed Recondo's point of view.

There is a new bit of tension when Janus, the leader, fails to come up with a definition of powerleveling. Recondo types in a fairly long comment- "Okay.,..don't mean to put you on the spot...this is a new discussion for me"- to slow things down, provide a little breathing room. It is after this utterance that the discussion moves toward resolution and agreement.

There has been a slight rend in the guild's moral universe. Through conversation, Sartor, Recondo, and Janus reweave the moral order and conclude the discussion by voicing agreement. The last statements in the exchange contain the words "accept" "yeah" and "yep," connoting agreement and consensus. New understandings have been reached, new shades of meaning articulated for the guild.

Let us return to Majimarg, who started the discussion. He was only making a joke, and has 
been silent since his first turn. But he has been paying attention. Majimarg asks what EQ is. He answers his own question and the discussion ends. Majimarg caps the discussion with a reappearance, just as Miggles capped the earlier discussion with a repetition. While such structure certainly does not characterize all conversations in World of Warcraft, it is notable to see it at all, and it would be interesting to do more in-depth analyses to see if such patterns emerge frequently or in certain kinds of conversations.

\section{Getting answers to questions -- fast}

How responsive is player culture in answering the questions of less experienced peers? We analyzed twelve hours of play in guild, party, local area, and general chat to find out how long players waited to receive answers to questions. Timestamped chat logs provide precise data. Timestamps are of the form e.g., 19:41:59.440. We rounded time-to-answer to the nearest second.

Questions cannot be automatically identified in transcripts; the transcripts must be read. We probably missed some questions in the hundreds of pages we analyzed. We identified 52 questions, eight of which were not answered in public chat (i.e., channels that are not private). It is possible that the questions were answered privately; it is not unusual for responders to whisper answers. The number of unanswered questions in our data is thus likely an overestimate. Our analysis is based on the remaining 44 questions which were answered in public chat.

Of these questions, answers were given on average after 32 seconds. Time-to-answer ranged from nine seconds to 184 seconds (3 minutes and 4 seconds). $25 \%$ of questions were answered in 13 seconds or less. Our responsiveness metric is calculated from the first answer received. As can be seen from the previous examples, answers to questions often continue as people converse about them.

Answering questions promptly and courteously is part of the learning culture of World of Warcraft (although of course not every question is answered in this way). In guild chat in particular players are watching to make sure guildmates get answers to their questions. Even in general chat where players do not necessarily know one another, answers are often supplied by friendly strangers.

\section{Discussion}

Our goal in this paper was to examine conversational activity in the zone of proximal development to investigate the nature of learning in World of Warcraft. We observed what Vygotsky predicted-that learners accomplish more with the aid of experienced peers than they could on their own. We described the contours of the learning experience in the ZPD as it unfolded in event-driven, erratic, spontaneous, emotionladen conversations closely tied to the context of activity. Through interviews with Acquaa, Coldnight, and other players we documented that the learning conversations that took place actually affected their ability to play, teaching them to play more effectively.

A surprising finding from our research was the emotionally inflected discourse in many learning conversations. Not every learning conversation involved emotion, but the drama, humor, and intimacy in conversations were unmistakable.

The zone of proximal development is generally taken to imply the acquisition of deeper understandings, new ways to integrate and make coherent concepts and ideas. It appears to us that the zone of proximal development is also about motivation and support as Vygotsky hinted [15]. The responsiveness players experience as they get fast answers to questions is part of what creates a supportive environment for learning. This seems to us to be part of the emotional aspect of the ZPD-positive encouragement, the avoidance of frustration, and a sense of moving forward.

Investigators such as Gee [4] and Steinkuehler [14] have emphasized that cognition in learning games is socially distributed, not an isolated individual activity. However, their account of distributed cognition, while insightful, still has a "rational actor" flavor, emphasizing problem solving, data gathering, and the ability to perform progressively more difficult tasks. In general, the concept of the distributed mind, while overcoming Cartesian isolation, is underdeveloped emotionally, exhibiting instrumentality but not emotional engagement. We observed that learning conversations in World of Warcraft are emotionally resonant with a rich array of emotions. Roth advocated that it is crucial to incorporate emotion into activitytheory based analyses and we have found that appropriate with our materials [12]. 
The emotions in learning conversations in World of Warcraft are generally lightweight, playful, and without lasting consequence. They occur in a virtual world with limited liability [see 2]. It is perhaps this very playfulness that makes emotion in learning conversations a useful resource for learning. Players are relaxed but engaged.

The presence of emotion in learning conversations is important because it suggests that despite the popularity of video games amongst youth, they are not a panacea for learning [see 12] because the emotion derives in part from the care players have for the game. They deeply enjoy the experiences of moving through the levels of play, gaining new equipment, exploring WoW's rich geographies, and the easy socializing characteristic of the game $[3,10]$. Learning conversations take place in a context in which players are already excited because of the many things they enjoy about the game.

We are not saying it is impossible to design compelling games for educational settings. But we observe that such games do not exist on any appreciable scale. Economically, entertainment titles are perceived to be more lucrative, with the exception of a few games such as Where in the World is Carmen San Diego?

However, human conversation always affords opportunities for emotion. Learning in conversation in games would likely generate more possibilities for emotional engagement than, say, online tutorials. During our interviews players mentioned that the competitive aspects of WoW heightened their emotions even to the point of increasing their heart rate.

This finding might suggest that teams of students playing educational games could compete in games that invoke knowledge associated with the traditional curriculum. As with any aspect of schooling, such games would be one part of the curriculum designed to complement other parts. For learning raw facts, games would seem to be a lively alternative to textbooks. Textbooks are still in wide use as teachers struggle to educate students on the basic facts of history, geography, science, and social studies which any educated person should know. Learning conversations among students in competitive social games might be as effective at helping students learn school content as are the conversations in World of Warcraft that impart game content.
A final observation is that the learning culture of World of Warcraft is constituted by players themselves through chat conversation. This culture has evolved such that learners can freely ask questions, they take it upon themselves to offer useful advice to less experienced peers, and moral knowledge is negotiated and shared. Game functionality such as chat channels supports this culture but in no way defines it. It is easy to imagine a very different culture in which it would not be appropriate to approach strangers to ask questions, players would not volunteer information, and introducing issues of game morality would be inappropriate. The credit for a learning culture that has developed in positive ways goes to the players of World of Warcraft and is a significant ingredient in the game's immense success.

\section{Conclusion}

Learning in conversation in World of Warcraft is erratic, spontaneous, contextual, and driven by small events. It is enlivened by playful, lightweight emotions. Such learning can support simple fact finding, the development of tactics and strategy, and the working out of a moral order.

In future research we will examine the extent to which other persistent online conversations may provide a curriculum with some structure. These resources include playerproduced online guides to developing particular classes as well as guides to successfully completing advanced quests undertaken at level 60. Players sometimes speak of "cookie-cutter" character development or quest management following such guides.

This intriguing pointer to a "cookie-cutter" approach to the game will be followed to discover whether learning in World of Warcraft may involve two distinct kinds of persistent conversations that enable learning-one completely unplanned and the other providing set-piece recipes for play. Notably, these persistent conversations are player-produced. Vygotsky himself, and most of his followers, developed accounts of the zone of proximal development in the context of formal schooling, emphasizing teachers as the key resource both in setting goals and providing aid as learners advanced. However, Vygotsky did not neglect more experienced peers as the other potential resource in the ZPD. In modern video games we find evidence of a zone of proximal development 
shaped by peers who voluntarily teach others in persistent conversations.

\section{Acknowledgments}

We would like to thank our informants who shared their experiences with us in interviews, casual chat, and sometimes game play. Anonymous reviewers provided helpful comments on the paper for which we are grateful.

\section{References}

[1] Blizzard Entertainment Inc. www.blizzard.com/press/060510.shtml

[2] Driskell, R. and Lyon, L. Are Virtual Communities True Communities? Examining the Environments and Elements of Community. City and Community 1 (2002), 373-390.

[3] Ducheneaut, N., Yee, N., Nickell, E., Moore, R. "Alone Together?" Exploring the social dynamics of massively multiplayer online games. Proceedings CHIO6, ACM Press, 2006

[4] Gee, J. What Video Games have to Teach Us about Learning and Literacy. Palgrave Macmillan, New York, 2003.

[5] Gherardi, S. Knowing in Organizations. London, Blackwell, 2006.

[6] Glaser, B. and Strauss, A. The Discovery of Grounded Theory. New York, Aldine, 1967.
[7] Lave, J. and Wenger, E. Situated Learning: Legitimate Peripheral Participation. Cambridge, Cambridge University Press, 1991.

[8] Merriam Webster Online.

[9] Meira, L. and Lerman, S. The Zone of Proximal Development as a symbolic space. 2001. London South Bank University, Social Science Research. www.lsbu.ac.uk/ahs/research/reports/stevelerman 1.pdf.

[10] Nardi, B. and Harris, J. Strangers and friends: Collaborative play in World of Warcraft. Forthcoming in Proceedings of the Conference on Computer-supported Cooperative Work, 2006.

[11] Roth, W.-M. (2005). Mathematical modeling 'in the wild': A case of hot cognition. In R. Lesh (ed.) Users of Mathematics: Foundations for the Future. Mahwah, NJ, Lawrence Erlbaum.

[12] Squire, K. Changing the game: What happens when video games enter the classroom? Innovate: Journal of Online Education 1 (2005).

[13] Stahl, G. Analyzing and designing the group cognitive experience. International Journal of Cooperative Information Systems 5 (2006).

[14] Steinkuehler, C. Cognition and Learning in Massively Multiplayer Online Games. Ph.D. Thesis, University of Wisconsin, 2005.

[15] Vygotsky, L. Mind and Society: The Development of Higher Mental Processes. Harvard University Press, Cambridge, 1978. 